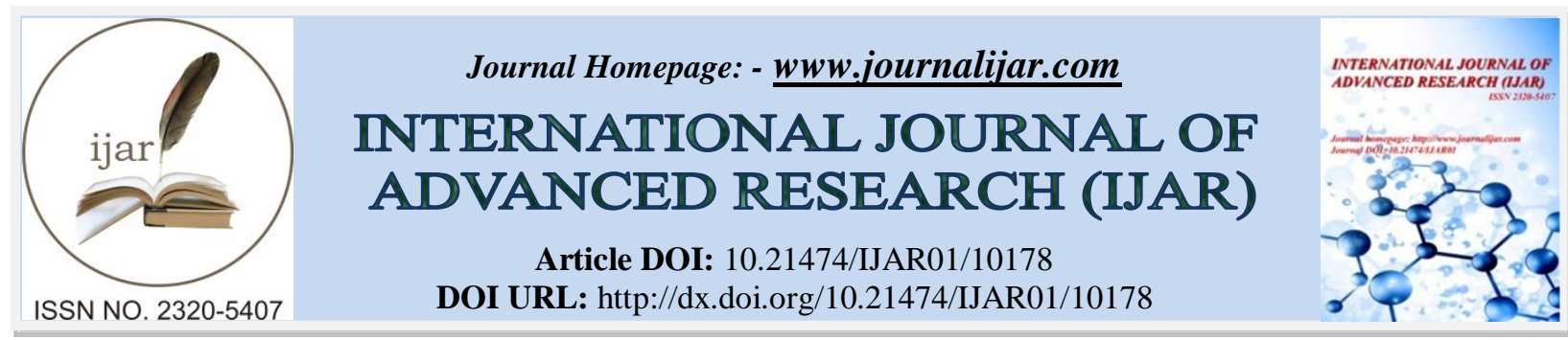

RESEARCH ARTICLE

\title{
WORMS PREVENTING EYE - RISE
}

\section{Dr. Shivani Nayak ${ }^{1}$ and Dr. Suhas Murali ${ }^{2}$}

1. Fellow in Phaco-Refractive surgery, Lions Super Specialty Eye Hospital, Bangalore.

2. Assistant Professor, Department of Neurology, Bangalore Medical College \& Research Institute.

\section{Manuscript Info}

\section{Manuscript History}

Received: 05 October 2019

Final Accepted: 07 November 2019

Published: December 2019

Key words:-

Superior rectus, Neurocysticercosis

\begin{abstract}
Diplopia is a commonly seen complaint in routine outpatient practice. However, unilateral diplopia in upward gaze in a middle aged gentleman, warrants a higher index of suspiscion of mechanical causes. We here present one such interesting cause of diplopia due to a cysticercal infection of the Superior Rectus muscle.
\end{abstract}

Copy Right, IJAR, 2019,. All rights reserved.

\section{Introduction:-}

A 48 year old gentleman presented with an insidious onset of diplopia on upward gaze since 1 month. He complained of reduced movement of the globe in upward direction on the right side. There was no associated history of trauma, fatigability, diurnal variation or ptosis in the same or the fellow eye. Other than his superior rectus on the right all other extra-ocular muscle movements were normal and full range. There was no evidence of $3^{\text {rd }}$ nerve involvement either. Hence we thought of a mechanical cause for the same and imaged the gentleman's orbit.

To our surprise we saw a lesion involving the entire length of the right superior rectus muscle which was hypointense in its core but taking up contrast along its margins brilliantly as shown. To find out what the lesion could be we did a fat-saturated orbit sequence in MRI which to our surprise showed a cystic lesion with the presence of a scolex in its posterior aspect (best seen in the sagittal image). To confirm we did a MRI-CISS 3D sequence which is a heavily T2 weighted image where CSF acts as an auto-contrast and this confirmed the presence of not just a scolex on the lesion but was also able to detect multiple lesions in the brain parenchyma, which were at varying stages in their clinical course. We confirmed the lesions were indeed due to Neurocysticercosis with the help of markedly elevated IgG antibodies in the CSF of the patient.

We present this case to highlight the importance of keeping infections as a top differential even for evaluation of ptosis, especially when there are no other risk factors.

Figure 1:- Shows a T1-post contrast image showing a hypointense lesion with brilliant contrast uptake involving the right superior rectus as shown.

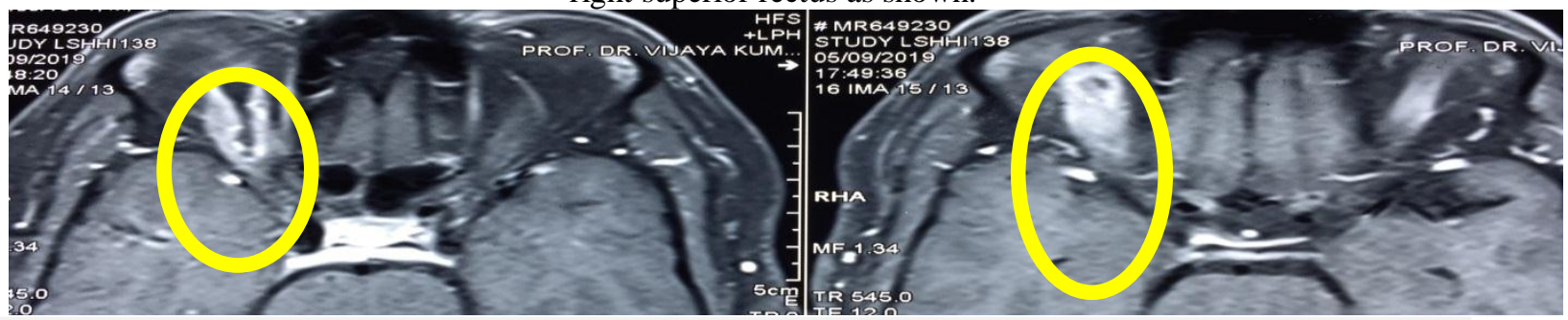

Corresponding Author:- Dr. Shivani Nayak

Address:- Fellow in Phaco-Refractive surgery, Lions Super Specialty Eye Hospital, Bangalore. 
Figure 2:- Shows the sagittal T1 post contrast images which show the involvement of the entire length of the superior rectus muscle as described.

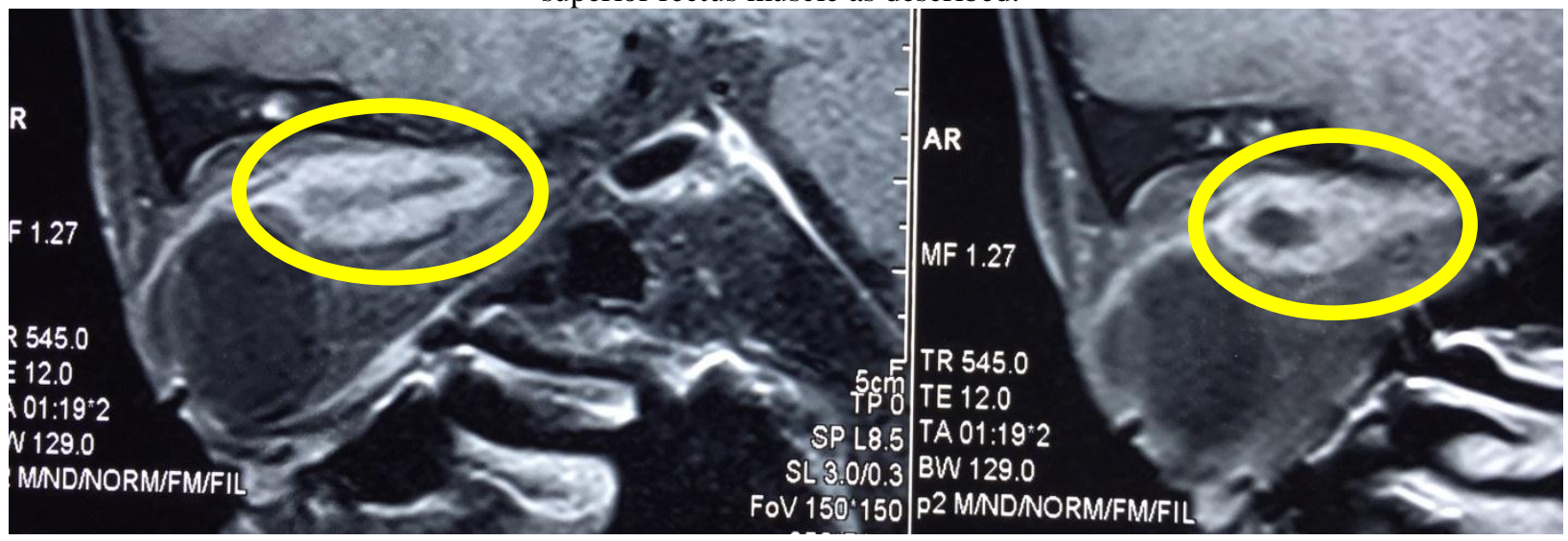

Figure 3:- Shows the sagittal fat-saturated sequence which shows the presence of a cystic lesion with the presence of a hypointense scolex (marked by red arrow) inside the hyperintense cavity which is the hallmark for a diagnosis of cysticercosis.

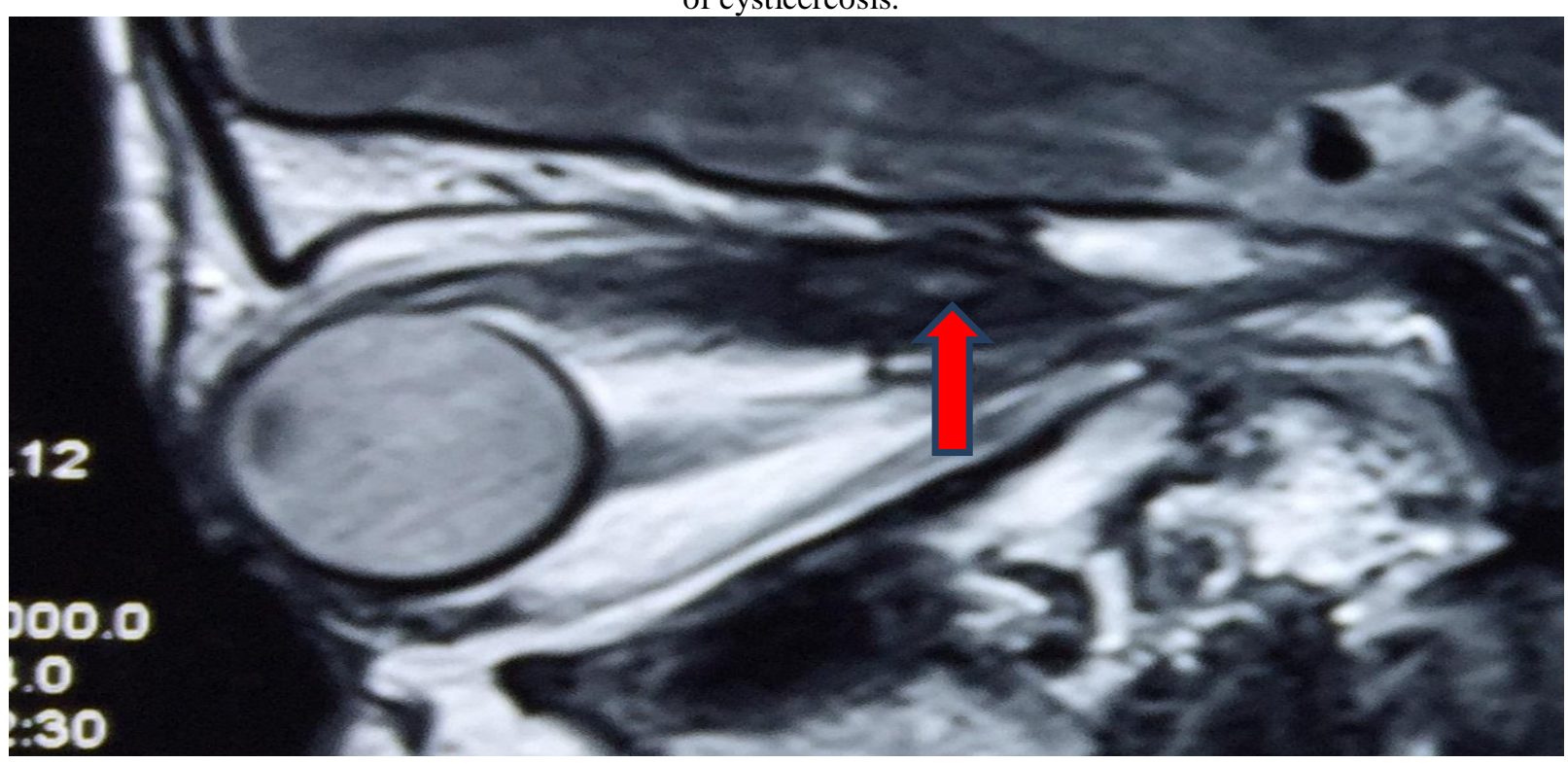

\section{References:-}

1. Amaral LL, Ferreira RM, da Rocha AJ, Ferreira NP. Neurocysticercosis: Evaluation with advanced magnetic resonance techniques and atypical forms. Top Magn Reson Imaging. 2005;16:127-44

2. Teitelbaum GP, Otto RJ, Lin M et al.. MR imaging of neurocysticercosis. AJR Am J Roentgenol 1989; 153(4):857-866.

3. Sotelo J, Guerrero V, Rubio F. Neurocysticercosis: a new classification based on active and inactive forms - a study of 753 cases. Arch Intern Med 1985;145(3):442-445

4. Barcelos, I. S., Mineo, J. R., Oliveira-Silva, D. A., Ferreira, M. S., Moura, L. P., Biondi, G. F. and Costa-Cruz, J. M. (2001). Detection of IgG in cerebrospinal fluid for diagnosis of neurocysticercosis: evaluation of saline and SDS extracts from Taenia solium and Taenia crassiceps metacestodes by ELISA and immunoblot assay. Tropical Medicine and International Health 6, 219-226. 